\title{
Resultados quirúrgicos y oncológicos de pacientes operados por cáncer de recto con asistencia robótica
}

\author{
Francisco López-Köstner ${ }^{1,2}$, Alessandra Cassana ${ }^{1}$, Alejandro Zárate C. ${ }^{1,3}$, Udo Kronberg ${ }^{1}$, \\ Claudio Wainstein G. ${ }^{1}$, Andrés Larach K. ${ }^{1}$ y Jorge Larach S. ${ }^{1}$
}

\section{Surgical and oncological results of patients operated for rectal cancer with robotic assistance}

\begin{abstract}
Aim: To analyze the surgical and oncological results of patients with rectal adenocarcinoma (RA) operated with robotic assistance. Materials and Method: Prospective cohort study, consecutive sample of patients between 2014-2017. Inclusion criteria: patients with primary RA, undergoing rectal resection, with robotic assistance with curative intention. Exclusion criteria: histology not adenocarcinoma. Evaluation of clinical-surgical data. Descriptive statistical analysis. Results: 37 patients were included; $20(54 \%)$ were men and average age was 58.7 years. The average distance from the anal margin to the distal edge of the tumor was $6.6 \mathrm{~cm}(2-12 \mathrm{~cm})$. Chemoradiotherapy (neoadyuvant) was indicated in 26 patients. The most frequent surgery was low anterior resection of the rectum and the average operating time was 266 minutes. Two conversions to laparotomy were performed. One or more complications were observed in 17 (45.9\%) patients, 9 of them were Clavien-Dindo III or IV, 5 patients $(13 \%)$ were reoperated. There were no blood transfusions and no postoperative mortality. The average postoperative hospital stay was 9.6 days (3-34). The average of resected lymph nodes was 15 . Surgical margins were negative in all patients. Intestinal transit was restored in 28/32 (87.5\%) patients. The average follow-up was 21 months (1-56), the overall and disease-free survival was $100 \%$. Discussion and Conclusion: Proctectomy with robotic assistance has proved to be safe in terms of early surgical results and oncologic indicators of the surgical piece.

Key words: colorectal neoplasms; robotics; rectal cancer.
\end{abstract}

\section{Resumen}

Objetivo: Analizar los resultados quirúrgicos y oncológicos de pacientes con adenocarcinoma de recto (AR) operados con asistencia robótica. Materiales y Método: Cohorte prospectiva entre 2014-2019. Criterios de inclusión: pacientes con AR primario, sometidos a una resección de recto con asistencia robótica con intención curativa. Criterios de exclusión: histología no adenocarcinoma. Evaluación de datos clínico-quirúrgicos. Análisis estadístico descriptivo. Resultados: Se incluyeron 37 pacientes; 20 (54\%) fueron hombres y la edad promedio fue 58,7 años. La distancia promedio desde el margen anal al borde distal del tumor fue $6,6 \mathrm{~cm}$ (i: $2-12 \mathrm{~cm}$ ). La quimiorradioterapia (neoadyuvancia) se indicó en 26 pacientes. La cirugía más frecuente fue la resección anterior baja de recto y el tiempo operatorio promedio fue $266 \mathrm{~min}$. Se realizaron dos conversiones a laparotomía. Una o más complicaciones se observaron en 17 (45,9\%) pacientes, 9 de ellos fueron Clavien-Dindo III o IV y se reoperaron 5 pacientes (13\%). No hubo transfusiones sanguíneas ni mortalidad posoperatoria. La estancia hospitalaria postoperatoria promedio fue 9,6 días (i: 3-34 d). El promedio de linfonodos resecados fue 15 (i 4-45). Los márgenes quirúrgicos fueron negativos en todos los pacientes. Se restituyó el tránsito intestinal en 28/32 (87,5\%) pacientes. El promedio de seguimiento fue 21 meses (1-56), la sobrevida global y libre de enfermedad fue $100 \%$. Discusión y Conclusión: La proctectomía con asistencia robótica ha demostrado ser segura en términos de resultados quirúrgicos tempranos y en criterios oncológicos de la pieza operatoria.

Palabras clave: neoplasias colorrectales; robótica; cáncer de recto.
Unidad de Coloproctología. Clínica Las Condes. Santiago, Chile.

${ }^{2}$ Facultad de Medicina, Universidad de Chile. Santiago, Chile. 3Departamento de Cirugía. Universidad Finis Terrae. Santiago, Chile.

Recibido 2020-07-17 y aceptado 2020-08-23

Correspondencia a: Dr. Francisco López-Köstner flopez@cliniclascondes.cl 


\section{Introducción}

El adenocarcinoma colorrectal se encuentra entre las primeras tres causas de mortalidad por cáncer a nivel mundial ${ }^{1}$, de ellos alrededor del $28 \%$ al $35 \%$ es por cáncer de recto ${ }^{1,2}$. La mortalidad en Chile ha ido en aumento ${ }^{3,4}$, posicionándose como la cuarta causa de muerte por enfermedades neoplásicas 5 .

El recto es el segmento final del tracto gastrointestinal, y generalmente, se define como los últimos $15 \mathrm{~cm}$ desde el margen anal, lo que corresponde a la zona del promontorio aproximadamente como límite proximal. Los tumores de recto por sobre la reflexión peritoneal, han demostrado comportarse como un cáncer de sigmoides ${ }^{6,7}$. Por otra parte, el cáncer de recto localizado por debajo de la reflexión peritoneal, que corresponden a los $10 \mathrm{~cm}$ del margen anal aproximadamente, es un ambiente anatómico más estrecho, con proximidad a estructuras como vagina, vesículas seminales, próstata, vasos pélvicos, plexos nerviosos, entre otros ${ }^{6}$; espacio en el cual el abordaje quirúrgico se convierte en un desafío para el cirujano.

El tratamiento del cáncer de recto ha sido tradicionalmente la cirugía, la cual se realiza vía laparotomía o mínimamente invasiva, clásicamente esta última con acceso laparoscópico ${ }^{8}$. La cirugía colorrectal mínimamente invasiva ha demostrado presentar diversas ventajas sobre el abordaje abierto ${ }^{9-11}$. Actualmente, el uso de laparoscopía es considerado el estándar de tratamiento en resecciones colorrectales electivas ${ }^{12,13}$, ya que se han demostrado mejores resultados postoperatorios en cuanto a una menor morbilidad, una recuperación funcional más rápida y una estadía postoperatoria más abreviada ${ }^{2,8,14-18}$. Asimismo, los resultados anátomo-patológicos de la pieza operatoria y la seguridad oncológica a corto plazo han evidenciado ser equivalentes a los alcanzados en cirugía abierta ${ }^{11,15,17,19}$.

La excepción a esta regla es la cirugía del recto extraperitoneal, ya que los instrumentos utilizados presentan limitaciones. De hecho, en los últimos años se han publicado dos trabajos prospectivos aleatorios multicentricos en los cuales no se ha logrado establecer la "no inferioridad" del acceso laparoscópico ${ }^{20,12}$. Es aquí donde debiera haber una ventaja para la cirugía asistida por robot. En los últimos años se ha introducido el abordaje de patologías intraabdominales vía robótica, a través del sistema da Vinci, desde su aprobación por la Food and Drug Administration (FDA) para cirugía abdominal en el año $2000^{22}$. En Chile, la primera cirugía robótica se llevó a cabo en el año 2009 para el manejo de cáncer de próstata en Clínica Las Condes, en junio de 2014 se realizó la primera cirugía robótica colorrectal.

En cuanto a los resultados de esta técnica en patologías neoplásicas, se describe su factibilidad y sus potenciales ventajas, en especial en el recto medio y distal ${ }^{23}$, zonas en las cuales mejoraría la disección por un mejor alcance y maniobrabilidad de los instrumentos en una plataforma tridimensional $^{1,2,9,10,16,17,19,24-26}$.

Los resultados oncológicos del abordaje robótico han sido descritos de forma favorable, siendo similares a los del abordaje laparoscópico ${ }^{27}$.

En este contexto, el presente trabajo describe los resultados de una serie de pacientes consecutivos con AR que fueron sometidos a una proctectomía con asistencia robótica, con el objetivo de analizar los resultados quirúrgicos y oncológicos a corto plazo.

\section{Materiales y Método}

\section{Diseño de estudio}

Se llevó a cabo un estudio de cohorte, prospectivo, analítico, transversal para evaluar los resultados oncológicos de pacientes operados de cáncer de recto con asistencia robótica con intención curativa entre los años 2014 y 2019. Los pacientes fueron operados por cirujanos de la Unidad de Coloproctología de la Clínica las Condes, Santiago de Chile.

La población de estudio correspondió a pacientes con diagnóstico preoperatorio de adenocarcinoma de recto; preferentemente, se seleccionaron para una proctectomía con asistencia robótica (Da Vinci SI) aquellos pacientes con un tumor extraperitoneal $y / u$ obesos y/o pelvis estrecha. Se excluyeron a todos aquellos cuyo diagnóstico histológico haya sido distinto al adenocarcinoma.

En los pacientes en quienes se decidió uso de neoadyuvancia, esta consistió en protocolo de radiación de 45 Gy dividida en 25 fracciones seguido de una dosis adicional de 5,4 Gy para el área tumoral, asociado a fluoropirimidinas vía oral o endovenosa, durante todos los días de radioterapia. La capecitabina se usó a dosis de $825 \mathrm{mg} / \mathrm{m}^{2}$ dos veces al día y el fuoruracilo se usó a dosis de $225 \mathrm{mg} / \mathrm{m}^{2}$ en infusión continua, ambos durante todos los días de radioterapia.

Se registraron datos clínicos, quirúrgicos, de anatomía patológica y del seguimiento en una base de datos aprobada por el comité de ética de la institución. Los eventos adversos postquirúrgicos 
ARTí́CULO ORIGINAL

se analizaron según la clasificación de ClavienDindo ${ }^{28}$. Se llevó a cabo un análisis descriptivo de las variables numéricas y categóricas empleando medias, desviaciones estándar y proporciones, respectivamente.

\section{Resultados}

En el periodo mencionado, se operaron 37 pacientes con AR vía robótica. De ellos, 20 (54\%) fueron hombres y la edad promedio fue de 58,7 años. Se realizó terapia neoadyuvante a 26 pacientes (70\%). Las características demográficas y clínicas generales se describen en la Tabla 1.

Respecto a las características del tumor en el preoperatorio, todos fueron adenocarcinomas rectales y la distancia promedio desde el margen anal al borde distal del tumor fue $6,6 \mathrm{~cm}(2-12 \mathrm{~cm})$.

Se realizaron 37 cirugías robóticas:

- 4 resecciones abdominoperineales.

- 14 resecciones anteriores bajas.

- 13 resecciones anteriores ultrabajas.

- 6 resecciones ultrabajas interesfintéricas.

De las cirugías en las que se realizó ileostomía en asa (32), se restituyó el tránsito intestinal a 28 pacientes $(87,5 \%)$. El tiempo operatorio medio fue de $266 \mathrm{~min}$. No se registraron transfusiones sanguíneas en el perioperatorio. Se convirtieron dos pacientes $(5,4 \%)$, debido a mala visualización y presencia de adherencias, respectivamente (Tabla 2).

En cuanto a los eventos adversos postquirúrgicos, se evidenciaron una o mas complicaciones en $17(46 \%)$ pacientes, las más frecuentes fueron la filtración de anastomosis, el íleo posoperatorio y la infección de sitio operatorio profundo, entre otras. De ellos, 7 pacientes fueron Clavien-Dindo III o IV y 5 fueron reoperados (13\%).

La estancia hospitalaria postquirúrgica media fue 9,6 días (3-34). No hubo mortalidad postoperatoria a 30 días (Tabla 3 ).

En cuanto al análisis anátomo-patológico de la pieza operatoria, el promedio de ganglios resecados fue de 15 . De los 37 pacientes, en 11 se evidenció compromiso linfonodal en la pieza quirúrgica (30\%). Los márgenes proximal, distal y circunferencial fueron negativos en todos los pacientes (Tabla 4).

El promedio de seguimiento fue de 21 meses (1-56) sin observarse recurrencia locorreguinal; la sobrevida global y libre de enfermedad fue $100 \%$.
Tabla 1. Características demográficas y clínicas de la población

\begin{tabular}{|c|c|c|c|}
\hline \multicolumn{2}{|l|}{ Características } & $\mathbf{n}$ & $\%$ \\
\hline \multirow[t]{2}{*}{ Sexo } & Femenino & 17 & 36 \\
\hline & Masculino & 20 & 54 \\
\hline Edad & $\begin{array}{l}\text { Media; desviación estándar } \\
\text { Intervalo }\end{array}$ & \multicolumn{2}{|c|}{$\begin{array}{l}58,7 ; 4,96 \\
{[35-79]}\end{array}$} \\
\hline \multirow[t]{2}{*}{ Índice de masa corporal } & Media & \multicolumn{2}{|c|}{26,5} \\
\hline & Intervalo & \multicolumn{2}{|c|}{$[19,3-39,8]$} \\
\hline \multirow[t]{8}{*}{ Antecedentes médicos } & Hipertensión arterial & 9 & 24,3 \\
\hline & Diabetes mellitus & 1 & 2,70 \\
\hline & Enfermedad cardiovascular & 3 & 8,1 \\
\hline & Dislipidemia & 5 & 13,5 \\
\hline & Obesidad & 7 & 18,9 \\
\hline & Otros cánceres* & 4 & 10,8 \\
\hline & Enfermedad diverticular & 2 & 5,4 \\
\hline & Asma & 2 & 5,4 \\
\hline Antecedentes quirúrgicos & Cirugía abdominal previa & 16 & 43,2 \\
\hline Antecedentes familiares & Cáncer colorrectal & 5 & 13,5 \\
\hline \multirow[t]{4}{*}{ Neoadyuvancia } & Total de pacientes & 26 & 70,3 \\
\hline & QT & 4 & 15,4 \\
\hline & RT & 5 & 19,2 \\
\hline & $\mathrm{QT}+\mathrm{RT}$ & 17 & 65,38 \\
\hline
\end{tabular}

*Cáncer de cérvix, útero y próstata. ${ }^{\dagger}$ Enfermedad pulmonar obstructiva crónica. *Enfermedad por reflujo gastroesofágico.

Tabla 2. Características quirúrgicas de la población

\begin{tabular}{|llcc|}
\hline Características & & n & \% \\
Tipo de cirugía & Resección abdominoperineal & 4 & 10,8 \\
& Resección anterior baja & 14 & 37,8 \\
& Resección anterior ultrabaja & 13 & 35,1 \\
& Resección anterior ultrabaja interesfintérica & 6 & 16,2 \\
Anastomosis & Colorrectal & 21 & 63,6 \\
& Coloanal & 12 & 36,4 \\
Ileostomía en asa & Total & $32 *$ & 86,5 \\
& Restitución intestinal & 28 & 87,5 \\
ASA & I & 10 & 27 \\
& II & 27 & 73 \\
Tiempo operatorio & Media; desviación estándar & $266 ; 101,9$ \\
& Intervalo & {$[165-506]$} \\
Conversión & Total & 2 & 5,4 \\
& Visualización inadecuada & 1 & 2,7 \\
\cline { 2 - 2 } & Síndrome adherencial & 1 & 2,7 \\
\hline Transfusiones sanguíneas & 0 & 0 \\
\hline
\end{tabular}

*No se incluyen las cuatro resecciones abdominoperineales y un paciente sometido a resección anterior baja al que se realizó colostomía terminal debido al compromiso tumoral. 
Tabla 3. Características posquirúrgicas de la población

\begin{tabular}{|llrr|}
\hline Características & & $\mathbf{n}$ & \multicolumn{1}{c}{$\%$} \\
Inicio de vía oral & Días (media; intervalo) & 2,$1 ;[1-11]$ \\
Soporte nutricional & & 5 & 13,5 \\
Cuidados intensivos & & 5 & 13,5 \\
Estancia hospitalaria & Total en días (media; intervalo) & 9,$6 ;[2-34]$ \\
& Postquirúrgica en días (media; intervalo) & 9,$6 ;[3-34]$ \\
Pacientes con complicaciones postquirúrgicas & 17 & 45,9 \\
Clavien-Dindo & I & 5 & 29,4 \\
& II & 3 & 17,5 \\
& IIIa & 2 & 11,8 \\
& IIIb & 6 & 35,3 \\
Causas & IV & 1 & 5,9 \\
& Infección de sitio operatorio profundo & 4 & 23,5 \\
& Infección de sitio operatorio órgano espacio & 1 & 5,9 \\
& Íleo postoperatorio & 5 & 29,4 \\
& Filtración de anastomosis & 6 & 35,3 \\
& Hematoma intraperitoneal & 2 & 11,8 \\
Reintervenciones quirúrgicas* & 1 & 5,9 \\
Mortalidad & Neumonía & 1 & 5,9 \\
\hline & Retención urinaria aguda & 5 & 13 \\
\hline
\end{tabular}

*lavado y drenaje laparoscópico.

Tabla 4. Características de pieza operatoria

\begin{tabular}{|llcc}
\hline Características & & $\mathbf{n}$ & $\mathbf{\%}$ \\
\hline $\mathrm{N}^{\mathrm{o}}$ de ganglios resecados & (Media; intervalo) & 15,$1 ;[4-45]$ \\
& Positivos & $11^{*}$ & 29,7 \\
Márgenes quirúrgicos $^{\dagger}$ & Radial (media; intervalo) & $16,4 ; ; 1-170]$ \\
& Proximal (media; intervalo) & $65,9 * ; 10-242]$ \\
& Distal (media; intervalo) & $18,4^{\ddagger} ;[1-64]$ \\
Invasión tumoral & pTis & 6 & 16,2 \\
& pT1 & 1 & 2,7 \\
& pT2 & 14 & 37,8 \\
& pT3 & 16 & 43,2
\end{tabular}

"Número de pacientes con ganglios positivos. "Negativos en todos los pacientes. Distancia en mm. casos, la cirugía laparoscópica se ha visto limitada debido a la complejidad y estrechez anatómica de la pelvis, más aún en pacientes de sexo masculino y obesidad ${ }^{16,24}$.

En esta serie de 37 pacientes con adenocarcinoma rectal, 35 (94,6\%) tuvieron un tumor ubicado entre el margen anal y los $10 \mathrm{~cm}$ proximales a este, los cuales fueron operados con asistencia robótica presentando resultados quirúrgicos similares a los reportados respecto al abordaje laparoscópico y acceso abierto.

La laparoscopía en la pelvis, específicamente en la disección rectal de los últimos 5 centímetros del recto, se ha visto limitada frente a la cirugía abierta, debido a dificultades técnicas de los instrumentos no articulados en una plataforma bidimensional ${ }^{17,29-31}$. El ensayo clínico controlado MRC ROLARR demostró una diferencia, si bien no significativa, en la positividad del margen circunferencial de un $6 \%$ vía laparoscópica versus un 5\% vía robótica ${ }^{27}$.

La cirugía robótica surge como una alternativa para la disección y resección oncológica de tumores rectales inferiores, al contar con instrumentos articulados con amplios rangos de movimientos bajo una plataforma tridimensional con mejor visualización y magnificación de la imagen ${ }^{2,9,14-17,19,27}$. Esto permite mejorar la precisión del cirujano durante la disección en espacios estrechos, a través de la identificación del plexo hipogástrico inferior para la preservación de nervios autonómicos, uréteres y vasos gonadales ${ }^{32}$, lo cual permitiría optimizar la excisión total del mesorrecto en la resección de tumores rectales ${ }^{11,17,19,24,32}$.

En cuanto a los resultados quirúrgicos encontrados en este estudio, el tiempo operatorio medio fue de $266 \mathrm{~min}$, valor que se encuentra dentro del rango reportado en investigaciones previas, que oscila entre 180 a $360 \min ^{10,24,33}$. Se registra un mayor tiempo operatorio en la cirugía robótica versus laparoscópica ${ }^{15}$, con una diferencia media de 37,5 a 58 min que se atribuye al docking de la consola, el posicionamiento del instrumental y la curva de aprendizaje $^{15,33}$. No obstante, como en la introducción de toda técnica quirúrgica, se señala que con la mejora de la curva de aprendizaje se reduce el tiempo operatorio a largo plazo ${ }^{24,27,34}$.

Respecto a las pérdidas sanguíneas, no se registraron transfusiones en el perioperatorio. Nuestro hallazgo concuerda con lo presentado en la literatura, en la que se describe una escasa pérdida sanguínea intraoperatoria, además, se señala que esta es igual o relativamente menor en comparación a la cirugía laparoscópica ${ }^{2,9,11,15,17,19,23-26,31,32}$. Yamaguchi et al. ${ }^{33}$, y Katsuno et al. ${ }^{10}$, registran valores 
muy bajos $(10 \mathrm{~mL})$, mientras que en una revisión sistemática de 21 investigaciones se reporta un rango de pérdida de 16 a $400 \mathrm{~mL}$ vía robótica ${ }^{35}$. Prete et al. ${ }^{15}$, comparan el valor medio de $158,3 \pm 219,3$ $\mathrm{mL}$ en cirugía robótica versus $162,5 \pm 197,4 \mathrm{~mL}$ en laparoscópica ${ }^{14-16,25-27,34}$. Asimismo, en la mayoría de los estudios no hubo necesidad de transfusión sanguínea ${ }^{10,14,16,25,27}$.

La tasa de conversión a cirugía abierta fue de $5,4 \%$ debido a mala visualización y a la presencia de adherencias. La tasa de conversión reportada en el estudio clínico aleatorizado ROLARR fue de $12,2 \%$ en cirugías laparoscópicas respecto a $8,1 \%$ de los pacientes operados por vía robótica ${ }^{27}$. Si bien el valor no fue estadísticamente significativo, múltiples estudios posteriores han puesto en evidencia que el uso de robot disminuiría la tasa de conversión ${ }^{9,11,23}$, encontrando valores de conversión de hasta $18 \%$ por laparoscopía versus 3,4\% por robótica ${ }^{17}$. Al analizar según el sexo, esta disminuye significativamente en pacientes de sexo masculino $(8,6 \%$ vía robótica versus $16,8 \%$ vía laparoscópica) $)^{11,17,24}$. Por otra parte, se ha demostrado una reducción significativa de la tasa de conversión en pacientes con obesidad ${ }^{1,10,11,15,27}$ y que fueron sometidos a resecciones anteriores bajas de recto ${ }^{29}$. Lo anterior es importante al considerar que, en nuestra serie, el $63 \%$ de los pacientes presentaron sobrepeso u obesidad.

En cuanto a los resultados postoperatorios, la estancia hospitalaria media fue de 9,6 días. No se reportan diferencias significativas entre el abordaje laparoscópico versus robótico ${ }^{11,26,31}$. Sin embargo, en algunos trabajos, la estancia hospitalaria resulta ser menor cuando el paciente se opera vía robótica ${ }^{19}$, con una variación de alta de uno o dos días antes que en los pacientes operados vía laparoscópica ${ }^{11,23}$.

Nueve pacientes tuvieron una o más complicaciones postoperatorias Clavien-Dindo III o IV, entre las que destacan la filtración de anastomosis e íleo postoperatorio como las causas más comunes. Se reconoce que la cirugía en pacientes con cáncer de recto es un procedimiento de morbilidad elevada en comparación con otras cirugías; nuestras cifras se enmarcan en lo reportado en la literatura ${ }^{15}$. No se han reportado diferencias significativas en el riesgo de dehiscencia de anastomosis entre ambos abordajes quirúrgicos ${ }^{1,11}$.

No hubo mortalidad postoperatoria a 30 días, hecho que concuerda con la baja tasa reportada en la literatura, donde la mortalidad general a los 30 días es muy similar entre ambos grupos de pacientes (entre $0,58 \%$ y $0,59 \%)^{27,32}$. Asimismo, no se han establecido diferencias significativas de mortalidad en ambos grupos ${ }^{10}$.
Respecto del análisis anátomo-patológico, presentamos una media de ganglios resecados de 15 , valor que se encuentra en el rango reportado de 13 a 20 en diversos estudios ${ }^{2,15,19,23,31}$. Asimismo, de los 37 pacientes operados, 11 (30\%) presentaron linfonodos comprometidos, valor que supera el promedio reportado por Matsuyama et al. (15,6 a $20,1 \%)^{11,14,17}$. Esto puede verse influenciado, a su vez, por el efecto de la radioterapia ${ }^{17}$; en nuestro estudio, el 70,3\% de la población recibió neoadyuvancia. La importancia recae en que el número de ganglios resecados se relaciona con los resultados oncológicos. Si bien se registra un mayor número de ganglios recuperados en los pacientes operados con asistencia robótica ${ }^{2,23,26}$, este resultado no ha presentado significancia estadística.

Los márgenes quirúrgicos fueron negativos en todos nuestros pacientes. En ROLARR no se encontró superioridad de la vía robótica sobre la laparoscópica, presentando una positividad del margen de resección circunferencial (MRC) de 5,1\% versus $6,3 \%$ respectivamente ${ }^{27}$; resultado que es corroborado por diversas investigaciones posteriores ${ }^{2,15,19,23,31}$. Sin embargo, algunos metaanálisis reportan que la cirugía robótica contribuye a mejorar los resultados de esta variable así como a incrementar el margen distal alcanzado ${ }^{11,14,17}$. En el presente estudio encontramos una media de margen distal de $1,8 \mathrm{~cm}$. Melstrom et al. ${ }^{17}$, reconoce un extra significativo de 0,5 a $0,9 \mathrm{~cm}$ vía robótica. Esto es de vital importancia, pues corresponden a medidas de calidad de la resección total del mesorrecto y constituyen un factor pronóstico de recurrencia local ${ }^{2,23,26}$.

Aun cuando no es el objetivo de este trabajo, debemos señalar que a la fecha no se ha observado ninguna recurrencia local ni a distancia, por lo que la sobrevida global y libre de enfermedad fue del $100 \%$, valor que concuerda con lo reportado en la literatura previa, donde no existen diferencias significativas de esta variable entre ambos abordajes ${ }^{11,15,19}$. No obstante, el seguimiento de nuestros pacientes es menor a 3 años.

\section{Conclusión}

La proctectomía con asistencia robótica ha demostrado ser segura en términos de los resultados quirúrgicos tempranos y en los criterios oncológicos de la pieza operatoria. Consideramos que el abordaje robótico es de gran utilidad en pacientes seleccionados, sobre todo en aquellos con cáncer de recto extraperitoneal, con pelvis estrecha y obesos, debido a las limitaciones anatómicas que ofrece dicha región para la disección. 


\section{Responsabilidades éticas}

Protección de personas y animales. Los autores declaran que para esta investigación no se han realizado experimentos en seres humanos ni en animales.
Confidencialidad de los datos. Los autores declaran que en este artículo no aparecen datos de pacientes.

Conflictos de interés: no hay.

\section{Bibliografía}

1. De Lacy FB, Chadi SA, Berho M, Heald RJ, Khan J, Moran B, et al. The Future of Rectal Cancer Surgery: A Narrative Review of an International Symposium. Surgical Innovation 2018;25:525-35. DOI: $10.1177 / 1553350618781227$.

2. Xi-Yu Sun, Lai Xu, Jun-Yang Lu \& Guan-Nan Zhang: Robotic versus conventional laparoscopic surgery for rectal cancer: systematic review and metaanalysis. Minimally Invasive Therapy \& Allied Technologies 2019;28:135-42. DOI: $10.1080 / 13645706.2018 .1498358$.

3. Souza DLB, Jerez-Roig J, Cabral FJ, de Lima JRF, Rutalira MK, Costa JAG. Colorectal cancer mortality in Brazil: Predictions until the year 2025 and cancer control implications. Diseases of the Colon and Rectum 2014;57:1082-9. DOI: 10.1097/DCR.0000000000000186.

4. Zarate AJ, Alonso FT, Garmendia ML, López-Köstner F. Increasing crude and adjusted mortality rates for colorectal cancer in a developing South American country. Colorectal Disease 2013;15:47-51. DOI: 10.1111/j.14631318.2012.03110.x.

5. American Cancer Society. Colorectal Cancer Facts \& Figures 2020-2022. [Internet] American Cancer Society 2020 [citado Julio 14, 2020]. Disponible en https://www.cancer.org/content/dam/ cancer-org/research/cancer-facts-andstatistics/colorectal-cancer-facts-andfigures/colorectal-cancer-facts-andfigures-2020-22.pdf

6. López-Köstner F, Carrillo K, Zárate AJ, Brien AO, Ladrón de Guevara

D. Cáncer de recto: Diagnóstico, estudio y estadificación. Rev Chil Cir 2012;64:199-209. DOI: 10.4067/s071840262012000200016.

7. López-Kostner F, Lavery IC, Hool GR, Rybicki LA, Fazio VW. Total mesorectal excision is not necessary for cancers of the upper rectum. Surgery 1998;124:612-8. DOI: $10.1067 / \mathrm{msy} .1998 .91361$.

8. Lavery IC, López-Kostner F, Pelley RJ, Fine RM. Treatment of colon and rectal cancer. Surgical Clinics of North America 2000;80:535-69. DOI10.1016/S00396109(05)70200-0.

9. Ngu JCY, Kim SH. Robotic surgery in colorectal cancer: The way forward or a passing fad. Journal of Gastrointestinal Oncology 2019;10:1222-8. DOI: 10.21037/jgo.2019.04.01

10. Katsuno H, Hanai T, Masumori K, Koide Y, Ashida K, Matsuoka H, et al. Robotic Surgery for Rectal Cancer: Operative Technique and Review of the Literature. Journal of the Anus, Rectum and Colon 2020;4:14-24. DOI: 10.23922/jarc.2019037.

11. Hoshino N, Sakamoto T, Hida K, Sakai Y. Robotic versus laparoscopic surgery for rectal cancer: an overview of systematic reviews with quality assessment of current evidence. Surgery Today 2019;49:556-70. DOI: 10.1007/s00595-019-1763-y.

12. Senagore AJ. Adoption of Laparoscopic Colorectal Surgery: It Was Quite a Journey. Clinics in Colon and Rectal Surgery 2015;28:131-4. DOI: $10.1055 / \mathrm{s}$ 0035-1560040

13. Larson DW, Nelson H. Laparoscopic colectomy for cancer. Journal of Gastrointestinal Surgery 2004;8:636-42. DOI: 10.1016/j.gassur.2004.03.002.

14. Achilli P, Grass F, Larson DW. Robotic surgery for rectal cancer as a platform to build on: review of current evidence. Surgery Today 2020:1-5. DOI: $10.1007 /$ s00595-020-02008-4.

15. Prete FP, Pezzolla A, Prete F, Testini M, Marzaioli R, Patriti A, et al. Robotic Versus Laparoscopic Minimally Invasive Surgery for Rectal Cancer: A Systematic Review and Meta-analysis of Randomized Controlled Trials. Annals of Surgery 2018;267:1034-46. DOI: 10.1097/ SLA.0000000000002523.

16. Matsuyama T, Kinugasa Y, Nakajima Y,
Kojima K. Robotic-assisted surgery for rectal cancer: Current state and future perspective. Annals of Gastroenterological Surgery 2018;2:406-12. DOI: 10.1002/ ags3.12202.

17. Melstrom K. Robotic Rectal Cancer Surgery. Cancer Treatment and Research 2016;168:295-308. DOI: 10.1007/978-3319-34244-3_14.

18. Fazio VW, López-Kostner F. Role of laparoscopic surgery for treatment of early colorectal carcinoma. World Journal of Surgery 2000;24:1056-60. DOI: 10.1007/ s002680010145.

19. Park EJ, Cho MS, Baek SJ, Hur H, Min BS, Baik SH, et al. Long-term Oncologic Outcomes of Robotic Low Anterior Resection for Rectal Cancer: A Comparative Study With Laparoscopic Surgery. Annals of Surgery 2015;261:129 37. DOI 10.1097/sla.0000000000000613.

20. Fleshman J, Branda M, Sargent DJ, Boller AM, George V, Abbas M, et al. Effect of laparoscopic-assisted resection vs open resection of stage II or III rectal cancer on pathologic outcomes the ACOSOG Z6051 randomized clinical trial. Journal of the American Medical Association 2015;314:1346-55. DOI: 10.1001/ jama.2015.10529.

21. Stevenson ARL, Solomon MJ, Lumley JW, Hewett P, Clouston AD, Gebski $\mathrm{VJ}$, et al. Effect of laparoscopicassisted resection vs open resection on pathological outcomes in rectal cancer: The ALaCaRT randomized clinical trial. Journal of the American Medical Association 2015;314:1356-63. DOI: 10.1001/jama.2015.12009.

22. Pigazzi A, Ellenhorn JDI, Ballantyne GH, Paz IB. Robotic-assisted laparoscopic low anterior resection with total mesorectal excision for rectal cancer. Surgical Endoscopy and Other Interventional Techniques 2006;20:15215. DOI: 10.1007/s00464-005-0855-5.

23. Nozawa H, Watanabe T. Robotic surgery for rectal cancer. Asian Journal of 
Endoscopic Surgery 2017;10:364-71. DOI: 10.1111 /ases.12427.

24. Azman ZAM, Kim SH. A review on robotic surgery in rectal cancer. Translational Gastroenterology and Hepatology 2016;1:1-7. DOI: 10.21037/ $\operatorname{tgh} .2016 .03 .16$.

25. Phan K, Kahlaee HR, Kim SH, Toh JWT. Laparoscopic vs. robotic rectal cancer surgery and the effect on conversion rates: a meta-analysis of randomized controlled trials and propensity-score-matched studies. Techniques in Coloproctology 2019;23:221-30. DOI: 10.1007/s10151018-1920-0.

26. Esen E, Aytac E, Ağcaoğlu O, Zenger S, Balik E, Baca B, et al. Totally Robotic Versus Totally Laparoscopic Surgery for Rectal Cancer. Surgical Laparoscopy, Endoscopy and Percutaneous Techniques. 2018;28:245-9. DOI: $10.1097 /$ SLE.0000000000000552.

27. Jayne D, Pigazzi A, Marshall H, Croft J, Corrigan N, Copeland J, et al. Effect of robotic-assisted vs conventional laparoscopic surgery on risk of conversion to open laparotomy among patients undergoing resection for rectal cancer the ROLARR randomized clinical trial. Journal of the American Medical Association 2017;318:1569-80. DOI: 10.1001/jama.2017.7219.

28. Dindo D, Demartines N, Clavien PA. Classification of surgical complications: A new proposal with evaluation in a cohort of 6336 patients and results of a survey. Annals of Surgery 2004;240:205-13. DOI: 10.1097/01.sla.0000133083.54934. ae.

29. Mak TWC, Lee JFY, Futaba K, Hon SSF, Ngo DKY, Ng SSM. Robotic surgery for rectal cancer: A systematic review of current practice. World Journal of Gastrointestinal Oncology 2014;6:184. DOI: 10.4251/wjgo.v6.i6.184.

30. Corrigan N, Marshall H, Croft J, Copeland J, Jayne D, Brown J. Exploring and adjusting for potential learning effects in ROLARR: A randomised controlled trial comparing robotic-assisted vs. standard laparoscopic surgery for rectal cancer resection. Trials 2018;19:339. DOI: 10.1186/s13063-018-2726-0.

31. Ielpo B, Durán H, Diaz E, Fabra I, Caruso R, Malavé L, et al. Robotic versus laparoscopic surgery for rectal cancer: a comparative study of clinical outcomes and costs. International Journal of Colorectal Disease 2017;32:1423-9. DOI: 10.1007/s00384-017-2876-7.

32. Li L, Zhang W, Guo Y, Wang X, Yu H, Du B, et al. Robotic Versus Laparoscopic Rectal Surgery for Rectal Cancer: A MetaAnalysis of 7 Randomized Controlled Trials. Surgical Innovation 2019;26:497504. DOI: $10.1177 / 1553350619839853$.

33. Yamaguchi T, Kinugasa Y, Shiomi A, Kagawa H, Yamakawa Y, Furuatni A, et al. Short- and long-term outcomes of roboticassisted laparoscopic surgery for rectal cancer: results of a single high-volume center in Japan. International Journal of Colorectal Disease. 2018;33:1755-62. DOI: $10.1007 / \mathrm{s} 00384-018-3153-0$.

34. Kim MJ, Park SC, Park JW, Chang HJ, Kim DY, Nam BH, et al. Robotassisted Versus Laparoscopic Surgery for Rectal Cancer: A Phase II Open Label Prospective Randomized Controlled Trial. Annals of Surgery 2018;267:243-51. DOI: $10.1097 /$ SLA.0000000000002321.

35. Gorgun E, Ozben V, Costedio M, Stocchi L, Kalady M, Remzi F. Robotic versus conventional laparoscopic rectal cancer surgery in obese patients. Colorectal Disease 2016;18:1063-71. DOI: 10.1111/ codi. 13374 . 